\title{
Resistance Modulation Action, Time-Kill Kinetics Assay, and Inhibition of Biofilm Formation Effects of Plumbagin from Plumbago zeylanica Linn
}

\author{
Emmanuel B. A. Adusei, ${ }^{1}$ Reimmel K. Adosraku, ${ }^{1}$ James Oppong-Kyekyeku (D), \\ Cedric D. K. Amengor $\left(\mathbb{D},{ }^{2}\right.$ and Yakubu Jibira ${ }^{3}$ \\ ${ }^{1}$ Department of Pharmaceutical Chemistry, Faculty of Pharmacy and Pharmaceutical Sciences, College of Health Sciences, \\ Kwame Nkrumah University of Science and Technology, Kumasi, Ghana \\ ${ }^{2}$ Department of Pharmaceutical Chemistry, School of Pharmacy, University of Health and Allied Sciences, Ho, Ghana \\ ${ }^{3}$ Department of Pharmacology, Faculty of Pharmacy and Pharmaceutical Sciences, College of Health Sciences, \\ Kwame Nkrumah University of Science and Technology, Kumasi, Ghana \\ Correspondence should be addressed to Cedric D. K. Amengor; camengor@uhas.edu.gh
}

Received 29 August 2019; Accepted 4 November 2019; Published 26 November 2019

Academic Editor: Jean-Paul J Gonzalez

Copyright ( 2019 Emmanuel B. A. Adusei et al. This is an open access article distributed under the Creative Commons Attribution License, which permits unrestricted use, distribution, and reproduction in any medium, provided the original work is properly cited.

\begin{abstract}
Antimicrobial resistance (AMR) is a threat to the prevention and treatment of the increasing range of infectious diseases. There is therefore the need for renewed efforts into antimicrobial discovery and development to combat the menace. The antimicrobial activity of plumbagin isolated from roots of Plumbago zeylanica against selected organisms was evaluated for resistance modulation antimicrobial assay, time-kill kinetics assay, and inhibition of biofilm formation. The minimum inhibitory concentrations (MICs) of plumbagin and standard drugs were determined via the broth microdilution method to be 0.5 to $8 \mu \mathrm{g} / \mathrm{mL}$ and $0.25-128 \mu \mathrm{g} / \mathrm{mL}$, respectively. In the resistance modulation study, MICs of the standard drugs were redetermined in the presence of subinhibitory concentration of plumbagin $(4 \mu \mathrm{g} / \mathrm{mL})$, and plumbagin was found to either potentiate or reduce the activities of these standard drugs with the highest potentiation recorded up to 12-folds for ketoconazole against Candida albicans. Plumbagin was found to be bacteriostatic and fungistatic from the time-kill kinetics study. Plumbagin demonstrated strong inhibition of biofilm formation activity at concentrations of 128,64 , and $32 \mu \mathrm{g} / \mathrm{mL}$ against the test microorganisms compared with ciprofloxacin. Plumbagin has been proved through this study to be a suitable lead compound in antimicrobial resistance drug development.
\end{abstract}

\section{Introduction}

Occurrence of infectious diseases in recent times is attributed mostly to bacteria; a result of prominent resistance strains of organisms to available antibiotics. These strains include drug-resistant Shigella, multidrug-resistant tuberculosis (MDR-TB), multidrug-resistant Escherichia coli and Neisseria gonorrheae, methicillin-resistant Staphylococcus aureus, and extensively resistant Gram-negative organisms like Pseudomonas aeruginosa and Acinetobacter baumannii [1]. Antimicrobial resistance stems from multifactorial causes which include irrational drug use, failure to complete prescribed dosage, prolonged drug use, and therapy duration $[2,3]$. Infections become difficult to treat due to AMR, while surgical and other medical procedures become highrisk intervention causing disability, prolonged sickness, and death [4]. Other consequences of AMR include passage of antibiotic resistant diseases to others and increase in economic burden on families, societies, and healthcare systems [4]. Modulation of the activities of commercial antimicrobials by phytochemicals may reverse the mechanism of resistance developed by the organisms [5]. Synergism of conventional antibiotics is being researched into in recent times to establish possible candidates for the discovery and 
development of new drugs to tackle the menace of antimicrobial resistance [6].

Time-kill kinetics assays help understand interactions that exist between microbial strains and antimicrobial agents. The assay shows a concentration or time-dependent test effect of antimicrobial agents on strains of microorganisms. It determines antimicrobial agents as bacteriostatic/fungistatic or bactericidal/fungicidal [7]. Bacteria develop resistance to drugs in different ways including formation of biofilms, active efflux of drugs, drug inactivation caused by enzyme secretion, and drug target site alteration [5]. Biofilm formation reduces the penetrating abilities, and as such bacteria producing biofilms are not affected by the mode of action of antibiotics [8]. Biofilms refer to cells of microbes embedded in selfproduced matrix of extrapolymeric substances attached irreversibly to a surface. Biofilms constitute $65 \%$ of microbial infections, and bacteria living in them develop resistance to antimicrobial agents a thousand times than those existing in free-living forms (planktonic forms) [9]. Potential agents to be considered in antimicrobial resistant drug development should therefore demonstrate strong biofilm reduction or inhibition activity [9].

Plumbago zeylanica Linn (Plumbaginaceae) is a bushy and straggling perennial shrub that grows between $1.5-3.0 \mathrm{~m}$ in height. It is distributed throughout tropical Africa with collection of the plant reported in Senegal, Guinea, Sierra Leone, Liberia, Ghana, Togo, Nigeria, Zambia, and Equatorial Guinea. Its roots are used traditionally to treat tuberculosis, gonorrhoea, diarrhoea, syphilis, wounds, and rheumatic pains and swellings $[10,11]$. The major component of the roots of the plant is plumbagin (5-hydroxy-2-methyl-1,4-naphthoquinone), a yellowish pigment also found naturally in plants of the Droseraceae, Ebenaceae, Iridaceae, Ancestrocladaceae, Drosophyllaceae, and Nepenthaceae families [12]. It has been reported to possess several biological activities including anticancer, antioxidant, antibacterial, antifungal, anti-inflammatory, antimalarial, and antiparasitic [13-19]. Plumbagin isolated from Plumbago scandens was evaluated for antibacterial activity by Paiva et al. using the broth macrodilution method. It exhibited a minimum inhibitory concentration (MIC) of $1.56 \mu \mathrm{g} / \mathrm{mL}$ against Staphylococcus aureus [18].

The resistance modulation action, time-kill kinetics, and effects on biofilm formation of plumbagin isolated from Plumbago zeylanica plant were investigated in this study and are hereby reported as our findings.

\section{Materials and Methods}

2.1. Materials and Reagents. The reagents used included the following: ethyl acetate (BDH Chemicals, UK), petroleum ether [VWR chemicals, U.S.A], acetic acid (Needham Market Suffolk, UK), dimethyl sulfoxide (DMSO) [SigmaAldrich, U.S.A], 3-(4, 5-dimethylthiazol-2-yl)-2, 5 diphenyl tetrazolium bromide (MTT), silica gel (70:230 mesh size) [Merck, U.S.A], silica gel coated TLC plates [Merck, Germany], crystal violet, 70\% v/v ethanol [Fischer Scientific, U.K], ciprofloxacin [Sigma-Aldrich, U.S.A], ketoconazole
[Sigma-Aldrich, U.S.A], amoxicillin [Sigma-Aldrich, U.S.A], and ampicillin [Sigma-Aldrich, U.S.A].

2.2. Test Organisms. Pure cultures of Staphylococcus aureus (ATCC 25923), Escherichia coli (ATCC 25922), Pseudomonas aeruginosa (ATCC 27853), Klebsiella pneumoniae (ATCC 10031), and Candida albicans (ATCC 10231) were obtained from the Microbiology laboratory of the Department of Pharmaceutics, Faculty of Pharmacy and Pharmaceutical Sciences, Kwame Nkrumah University of Science and Technology (KNUST), Kumasi, Ghana.

2.3. Collection of Plant Material. The Plumbago zeylanica roots were collected from the Physic garden in the Faculty of Pharmacy and Pharmaceutical Sciences, Kwame Nkrumah University of Science, and Technology (KNUST) Kumasi, Ghana, in September 2018. The collected sample was authenticated by Mr. Clifford Osafo Asare, the horticulturist with the Department of Pharmacognosy, KNUST, and deposited in the Department's herbarium with a voucher specimen number of $(003 / 10 / 07)$.

2.4. Isolation of Plumbagin. The powdered roots $(2.4 \mathrm{~kg})$ of Plumbago zeylanica were extracted with ethyl acetate for 5 days, and the extract obtained was concentrated with rotary evaporator (Buchi, Switzerland/R-114) at a temperature of $40^{\circ} \mathrm{C}$ and controlled vacuum pressure, to obtain a dried mass. Plumbagin $(2.7 \mathrm{~g})$ was eluted with a mobile phase composition of ethyl acetate $(15 \% \mathrm{v} / \mathrm{v})$ in petroleum ether $(85 \% \mathrm{v} / \mathrm{v})$ from a silica gel column.

2.5. Confirmation of Isolate. The isolate's identity was established with melting point, 1D-NMR (proton and carbon-13), and 2D-NMR (COSY, HMBC, HSQC, and DEPT135) spectroscopy, with the support of infrared (IR) and ultraviolet-visible (UV-Vis) spectroscopy.

The melting point of the isolate was determined in triplicate with the Stuart melting point apparatus [UK/ R000105350].

The UV-spectrophotometer (Jenway, UK/7315) was calibrated in the range of 200 to $700 \mathrm{~nm}$ of wavelength, with methanol as blank. A quantity $(4 \mathrm{mg})$ of the isolate was dissolved completely in methanol and the UV-spectrum of the methanolic solution of the isolate subsequently determined.

The IR spectrum of the isolate was obtained using a Fourier-transform Infra-red (FTIR) (Bruker FTIR spectrometer) with the sample at a wavelength range of $4000 \mathrm{~cm}^{-1}$ to $400 \mathrm{~cm}^{-1}$.

Spectra for 1D-NMR (proton and carbon-13) as well as 2D-NMR techniques like COSY, HMBC, HSQC, and DEPT135 were obtained with the Bruker Biospin NMR spectrometer (Billerica, US/F/NMR/A 175). The sample was run using deuterated chloroform $\left(\mathrm{CDCl}_{3}\right)$ at a frequency of $500 \mathrm{MHz}$ and temperature of $298 \mathrm{~K}$. 
2.6. Determination of Minimum Inhibitory Concentrations of Plumbagin and Standard Drugs. The minimum inhibitory concentration (MIC) of plumbagin and the reference drugs (ciprofloxacin, amoxicillin, ampicillin, and ketoconazole) were determined with the broth microdilution method. The 96-well microtitre plates were singly filled with $100 \mu \mathrm{L}$ of double strength nutrient broth and subsequently with different concentrations of plumbagin and reference drugs ranging from 0.25 to $256 \mu \mathrm{g} / \mathrm{mL}$. Twenty microliters of $10^{6} \mathrm{cfu} / \mathrm{mL}$ of test organisms were added to each well. Selected wells were filled with nutrient broth only and nutrient broth and organisms only, to serve as negative and positive controls, respectively. The microtitre plates were then incubated at $37^{\circ} \mathrm{C}$ for $24 \mathrm{~h}$, after which $20 \mu \mathrm{L}$ of $1.25 \mathrm{mg} / \mathrm{mL}$ of 3-(4, 5-dimethylthiazol-2-yl)-2, 5-diphenyltetrazolium bromide (MTT) was introduced into each well and observations made for a purple coloration after $30 \mathrm{~min}$ of incubation, which signifies growth. The minimum concentrations of plumbagin and reference drugs that did not show any colour change in the wells were selected as the MIC. The experiment was carried out in triplicate [5].

2.7. Resistance Modulation Studies. The MICs of the antibiotics and antifungal were determined in the presence of subinhibitory concentration $(4 \mu \mathrm{g} / \mathrm{mL})$ of plumbagin using the broth microdilution technique. The 96-well microtitre plates wells were singly filled with $100 \mu \mathrm{L}$ of double-strength nutrient broth, appropriate volumes of different concentrations of the drugs, and $20 \mu \mathrm{L}$ of $10^{6} \mathrm{cfu} / \mathrm{mL}$ of test organisms. The plates were incubated for $24 \mathrm{~h}$ at $37^{\circ} \mathrm{C}$, after which $20 \mu \mathrm{L}$ of MTT was introduced to the wells, and the MICs were determined as the lowest concentration at which no growth was observed by colour change to purple [5, 20-22].

2.8. Time-Kill Kinetics Assay of Plumbagin. Time kill kinetics of plumbagin was carried out using a modified procedure described by Appiah et al. [23]. The organisms employed (Staphylococcus aureus ATCC 25923, Pseudomonas aeruginosa ATCC 27853, Escherichia coli ATCC 25922, and Candida albicans ATCC 10231) were subcultured and diluted to $0.5 \mathrm{McF}$ arland standard. Concentrations equal to MIC, twice the MIC, and four times the MIC of plumbagin were prepared and transferred into sterile broth in test tubes, after which an inoculum size of $1.0 \times 10^{6} \mathrm{cfu} / \mathrm{mL}$ of the standardized organisms was added and the test tubes incubated for $37^{\circ} \mathrm{C}$. Aliquots $(1.0 \mathrm{~mL})$ of the medium were taken at time intervals of $0,6,12,18,24$, and $30 \mathrm{~h}$ for bacteria and $0,6,12,30,36,48,54$, and $72 \mathrm{~h}$ for fungi and aseptically inoculated into nutrient agar in sterile Petri dishes. The agar was allowed to set, and the Petri dishes containing inoculum were incubated at $37^{\circ} \mathrm{C}$ for $24 \mathrm{~h}$. A control test was performed alongside for the organisms only. The colony-forming unit (cfu) of the test organisms was determined and the procedure performed in triplicate. A graph of $\log \mathrm{CFU} / \mathrm{mL}$ was plotted against time. The data obtained from the study were analysed using one-way ANOVA followed by Dunnett's post hoc test from Graph Pad Prism Version 5.10 for windows (Graph Pad Software Inc., San Diego, CA, USA) [23].
2.9. Formation of Bacteria Biofilm. Pure cultures of the four bacteria (Staphylococcus aureus ATCC 25923, Pseudomonas aeruginosa ATCC 27853, Klebsiella pneumoniae ATCC 10031, and Escherichia coli ATCC 25922) diluted to 0.5 McFarland standard in Mueller-Hinton broth were employed. Ten microliters $(10 \mu \mathrm{L})$ of the standardized cultures was added to $100 \mu \mathrm{L}$ of broth in wells of a 96-well microtitre plate and the plate incubated at $37^{\circ} \mathrm{C}$ for $24 \mathrm{~h}$. Planktonic cells were aspirated and the wells washed with sterile water to get rid of free floating bacteria after incubation. The bacteria biofilms formed in the wells were dried between $25^{\circ} \mathrm{C}$ to $28^{\circ} \mathrm{C}$ and stained with $0.1 \%$ crystal violet for $20 \mathrm{~min}$. The stain was washed with sterile water and dried. The stained bacteria biofilm stuck to the wall of the wells were reconstituted in ethanol/acetic acid (1:1), and the absorbance read at $595 \mathrm{~nm}$ with a multimode microtitre plate reader. The optical density (OD) of the sterile broth was subtracted from that of the biofilm formed to eliminate background absorbance. The determination was performed in triplicate [24].

2.10. Inhibition Effect of Plumbagin on Biofilm Formation. Plumbagin and reference drug ciprofloxacin were reconstituted in dimethyl sulfoxide (DMSO) $(1 \% \mathrm{v} / \mathrm{v})$. Two-fold dilutions were made to achieve a concentration range of 1 to $128 \mu \mathrm{g} / \mathrm{mL}$. Aliquots of $100 \mu \mathrm{L}$ of double-strength Mueller-Hinton broth was added to the 96-well microtitre plate and the sample solution (plumbagin and ciprofloxacin) added. Ten microliters of microorganisms ( $24 \mathrm{~h}$ broth culture diluted to $0.5 \mathrm{McF}$ arland standard and containing $10^{6}$ cells per $\mathrm{mL}$ ) was added, and the plate was incubated at $37^{\circ} \mathrm{C}$ for $24 \mathrm{~h}$. The planktonic cells were aspirated and the wells washed, dried, fixed, and stained after incubation. Absorbance was read at $595 \mathrm{~nm}$ and the optical density (OD) of the culture media control subtracted to obtain the inhibitory effects of plumbagin and ciprofloxacin. The determination was carried out in triplicate. The ability of the plumbagin and the reference drug (ciprofloxacin) to reduce the optical density (OD) compared to the negative control was considered the biofilm inhibitory activity [24]"

\section{\% biofilm inhibition \\ $=\frac{\text { optical density }(\mathrm{OD}) \text { of control }- \text { OD of treatment }}{\text { optical density }(\mathrm{OD}) \text { of control }} \times 100$.}

\section{Results and Discussion}

3.1. Characterization of Plumbagin. Plumbagin $(1.5 \mathrm{~g})$ was obtained (after recrystallization) as yellowish needle-like crystals with a melting point of $78-80^{\circ} \mathrm{C}$. The retardation factor $\left(R_{\mathrm{f}}\right)$ was obtained on a silica gel coated TLC plate developed with mobile phase of petroleum ether-ethyl acetate $(70 \%: 30 \%)$ as 0.82 .

The melting point of the isolated compound was obtained to be $78-80^{\circ} \mathrm{C}$. This closely agrees with $78-79^{\circ} \mathrm{C}$ reported for plumbagin in the literature [13]. The sharp and 
undepressed nature of the melting point range obtained for the isolate emphasizes the purity of the compound.

The UV-Vis spectrum of the isolate showed absorbance of 0.339 and 0.093 at wavelengths of $265 \mathrm{~nm}$ and $410 \mathrm{~nm}$, respectively, indicating the presence of an extended conjugated chromophore system (Figure 1).

The IR spectrum showed a broad band at $3293 \mathrm{~cm}^{-1}$ that pointed to the $\mathrm{OH}$ group on the carbon at position 5. Peaks seen at wavenumbers $1161.60 \mathrm{~cm}^{-1}$ and $1639.66 \mathrm{~cm}^{-1}$ point to the free and hydrogen-bonded carbonyls at positions 1 and 4 , respectively. Aromatic $\mathrm{C}=\mathrm{C}$ vibrations observed at $1605.48 \mathrm{~cm}^{-1}$ is attributed to the carbons at positions 2 and 3, 5 and 10, 6 and 7, and 8 and 9. The absorption seen at $3038.4 \mathrm{~cm}^{-1}$ revealed $-\mathrm{C}-\mathrm{H}$ stretches of aromatic $\mathrm{Sp}^{2}$ carbons (Figure 2).

The NMR data obtained further confirmed the isolated compound to be plumbagin. Chemical shifts and peak multiplicities obtained for the compound in the proton NMR analysis in deuterated chloroform (Table 1) bear similarities to the reported values in the literature [13]. The chemical shift integrals revealed a total of 8 protons which is in agreement with the molecular formulae of $\mathrm{C}_{11} \mathrm{H}_{8} \mathrm{O}_{3}$. The carbon-13 analysis of the compound in deuterated chloroform showed 11 carbons consistent with the molecular formula of plumbagin (Figure 1).

3.2. Minimum Inhibitory Concentration (MIC). Growth was seen in all the positive controls which indicated that the nutrient broth sustained growth of the organisms employed. No growth was observed in the negative controls, implying that the equipment used and environment did not introduce microorganisms into the nutrient broth. The minimum inhibitory concentration (MIC) of plumbagin and the standard antimicrobials used against the test organisms is shown in Table 2.

3.3. Resistance Modulation Studies. The resistance modulation study involved determination of MICs of standard antimicrobials in the presence of subinhibitory concentrations of the agent(s) under investigation. Subinhibitory concentration is half the MIC of the agent being investigated for modulation effect that ordinarily does not inhibit the growth of microorganisms. MICs of the antimicrobials were redetermined at the concentration range of $0.25-256 \mu \mathrm{g} / \mathrm{mL}$ in the presence of plumbagin's subinhibitory concentration of $4 \mu \mathrm{g} / \mathrm{mL}$ chosen from a range of $0.25-4 \mu \mathrm{g} / \mathrm{mL}$ at $37^{\circ} \mathrm{C}$ for $24 \mathrm{~h}$. Decrease in MIC obtained implied potentiation of activities and increase in MIC suggested decrease in activities of the antimicrobial agents against the test organisms. Ciprofloxacin's activity was potentiated by 2 -folds against Staphylococcus aureus, Pseudomonas aeruginosa, and Escherichia coli. The activity decreased by 2 -folds against Klebsiella pneumoniae. Activity of amoxicillin was potentiated by 2-folds, 2-folds, and 6-folds against Staphylococcus aureus, Escherichia coli, and Klebsiella pneumoniae, respectively. The activity was however decreased by 2 -folds against Pseudomonas aeruginosa. Ampicillin's activity was potentiated by 2-folds, 2-folds, and 6-folds against

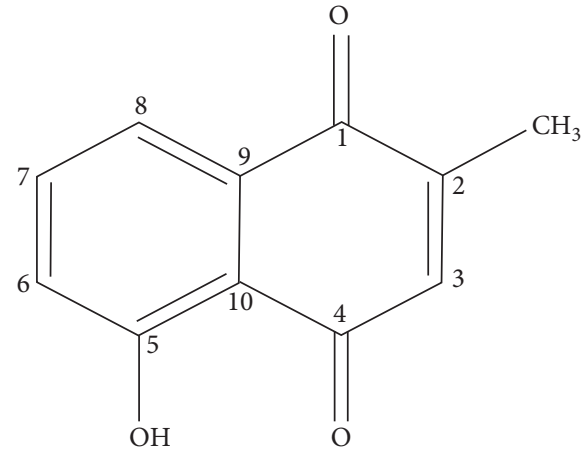

FIgURE 1: Chemical structure of plumbagin.

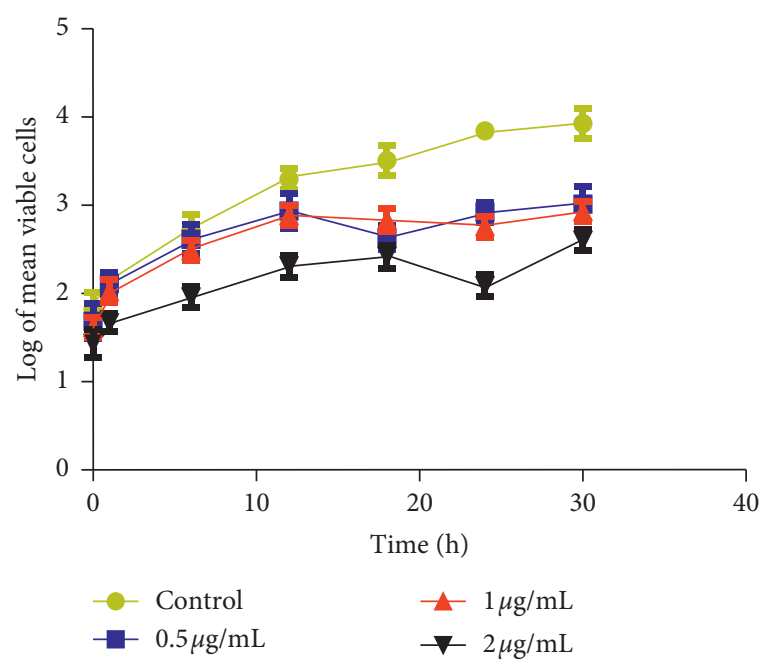

(a)

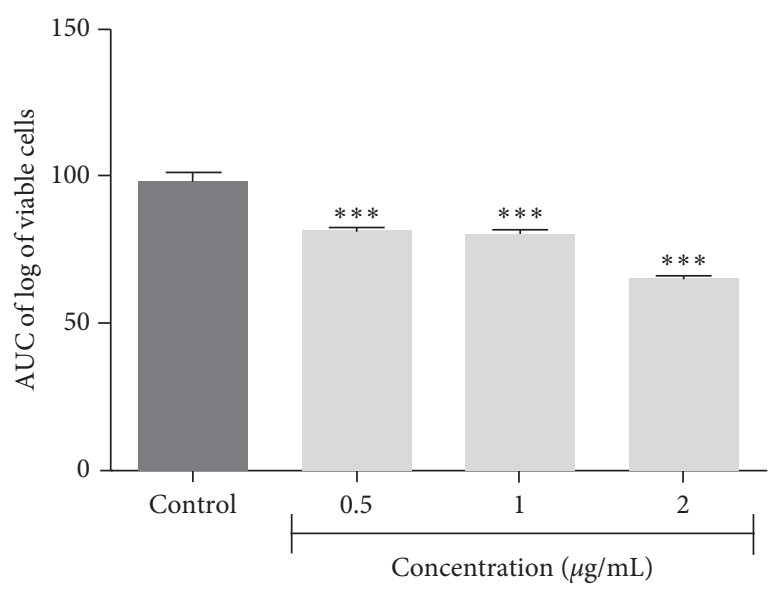

(b)

Figure 2: Time-kill kinetics of plumbagin against Staphylococcus aureus. (a) Time-kill kinetics curve and (b) AUC of time-kill kinetics. AUC: area under the curve. $n=3$; values are mean \pm SEM. ${ }^{* * *} p<0.0001$ (one-way ANOVA followed by Dunnett's post hoc test).

Staphylococcus aureus, Escherichia coli, and Klebsiella pneumoniae, respectively, and decreased by 2 -folds against Pseudomonas aeruginosa. Ketoconazole's activity was potentiated 
TABLE 1: MICs of standard antimicrobial drugs in the presence of subinhibitory concentration of plumbagin $(4 \mu \mathrm{g} / \mathrm{mL})$.

\begin{tabular}{|c|c|c|c|c|c|c|c|c|c|c|}
\hline \multirow{3}{*}{ Antimicrobials } & \multicolumn{10}{|c|}{$\operatorname{MIC}(\mu \mathrm{g} / \mathrm{mL})$} \\
\hline & \multicolumn{2}{|c|}{ S. aureus } & \multicolumn{2}{|c|}{ E. coli } & \multicolumn{2}{|c|}{ K. pneumoniae } & \multicolumn{2}{|c|}{ P. aeruginosa } & \multicolumn{2}{|c|}{ C. albicans } \\
\hline & MIC & Folds & MIC & Folds & MIC & Folds & MIC & Folds & MIC & Folds \\
\hline Ciprofloxacin & 0.5 & $2(+)$ & 0.25 & $2(+)$ & 2 & $2(-)$ & 0.25 & $2(+)$ & & \\
\hline Amoxicillin & 0.25 & $2(+)$ & 2 & $2(+)$ & 4 & $6(+)$ & 256 & $2(-)$ & & \\
\hline Ampicillin & 0.25 & $2(+)$ & 2 & $2(+)$ & 4 & $6(+)$ & 256 & $2(-)$ & & \\
\hline Ketoconazole & & & & & & & & & 4 & $12(+)$ \\
\hline
\end{tabular}

(+): reduction in MIC (increased activity). (-): increase in MIC (decreased activity).

TABLE 2: MICs of plumbagin and antimicrobials against test organisms.

\begin{tabular}{lccccc}
\hline Antimicrobials & & MIC $(\mu \mathrm{g} / \mathrm{mL})$ & P. aeruginosa & C. albicans \\
\hline Plumbagin & S. aureus & E. coli & K. pneumoniae & $\mathbf{2}$ & $\mathbf{0 . 5}$ \\
Ciprofloxacin & $\mathbf{0 . 5}$ & $\mathbf{8}$ & $\mathbf{1}$ & $\mathbf{1 2 8}$ \\
Amoxicillin & $\mathbf{0 . 5}$ & $\mathbf{4}$ & $\mathbf{3 2}$ & $\mathbf{1 2 8}$ \\
Ampicillin & $\mathbf{0 . 5}$ & $\mathbf{4}$ & $\mathbf{3 2}$ & $\mathbf{2 5 6}$ \\
Ketoconazole & $\mathbf{0 . 5}$ & & & \\
\hline
\end{tabular}

by 12 -folds against Candida albicans in the presence of subinhibitory concentration of plumbagin (Table 1).

3.4. Time-Kill Kinetics Assay of Plumbagin. The time-kill kinetics profile of the isolated plumbagin against Staphylococcus aureus showed a reduction in the number of viable cells between $18 \mathrm{~h}$ and $24 \mathrm{~h}$ accompanied by an increase up to $30 \mathrm{hrs}$ compared to the control (growth of organism without antimicrobial agents) (Figure 2). Time-kill kinetics profile of plumbagin against Escherichia coli at the test concentrations used showed a reduction in the number of viable cells between $12 \mathrm{~h}$ and $18 \mathrm{~h}$, followed by a gradual rise up to the $24 \mathrm{hr}$ and subsequent reduction up to the $30 \mathrm{~h}$ against its control (Figure 3). Plumbagin's time-kill kinetics profile against Pseudomonas aeruginosa at the various test concentrations showed a reduction in the number of viable cells between $12 \mathrm{~h}$ and $18 \mathrm{~h}$, remained constant till $24 \mathrm{~h}$, and followed by a gradual rise up to $30 \mathrm{~h}$ when compared to its control (Figure 4). Time-kill kinetics profile of plumbagin against Candida albicans at the test concentrations used showed a reduction in the number of viable cells between $30 \mathrm{~h}$ and $48 \mathrm{~h}$, followed by a gradual rise up to $54 \mathrm{~h}$ and subsequent reduction to $72 \mathrm{~h}$, compared to its control (Figure 5).

The antimicrobial effect of plumbagin was observed to be bacteriostatic against the test bacteria (Staphylococcus aureus, Escherichia coli, and Klebsiella pneumoniae) and fungistatic against the fungus (Candida albicans) used. The area under the curve (AUC) at the concentrations used showed significant reduction in the number of viable cells $(p<0.0001)$ compared to the negative controls (Figures 2-5).

3.5. Inhibition of Biofilm Formation Study. Plumbagin was assessed for its ability to either completely inhibit or reduce formation of bacteria biofilm using the concentration ranging from $1-128 \mu \mathrm{g} / \mathrm{mL}$. Ciprofloxacin was employed as

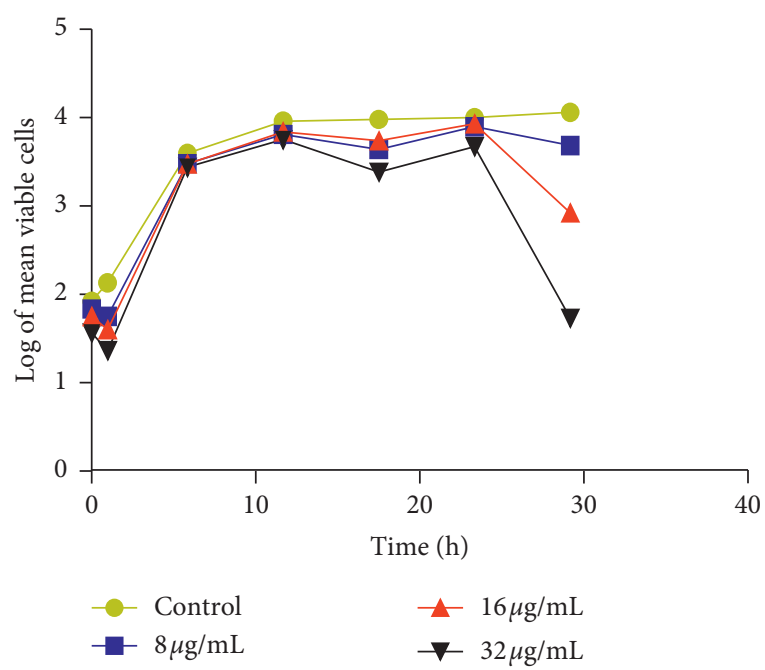

(a)

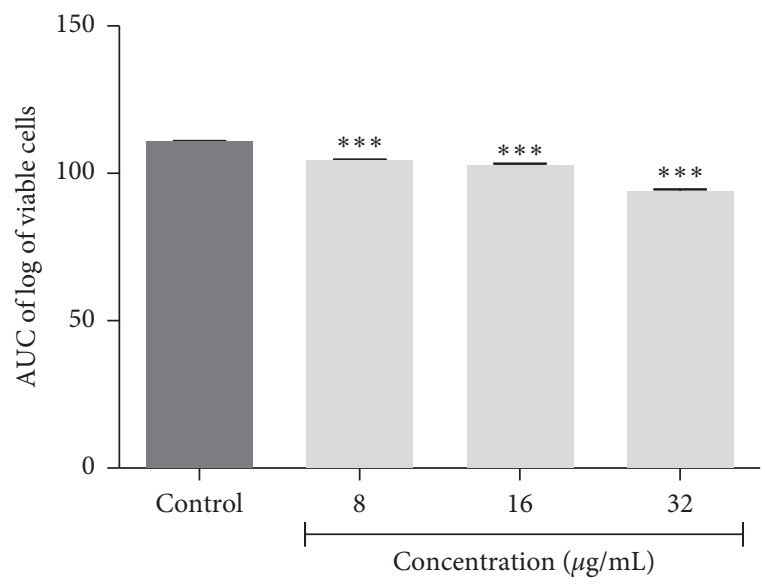

(b)

Figure 3: Time-kill kinetics of plumbagin against Escherichia coli. (a) Time-kill kinetics curve and (b) AUC of time-kill kinetics. AUC: area under the curve. $n=3$; values are mean \pm SEM. ${ }^{* * *} p<0.0001$ (one-way ANOVA followed by Dunnett's post hoc test). 


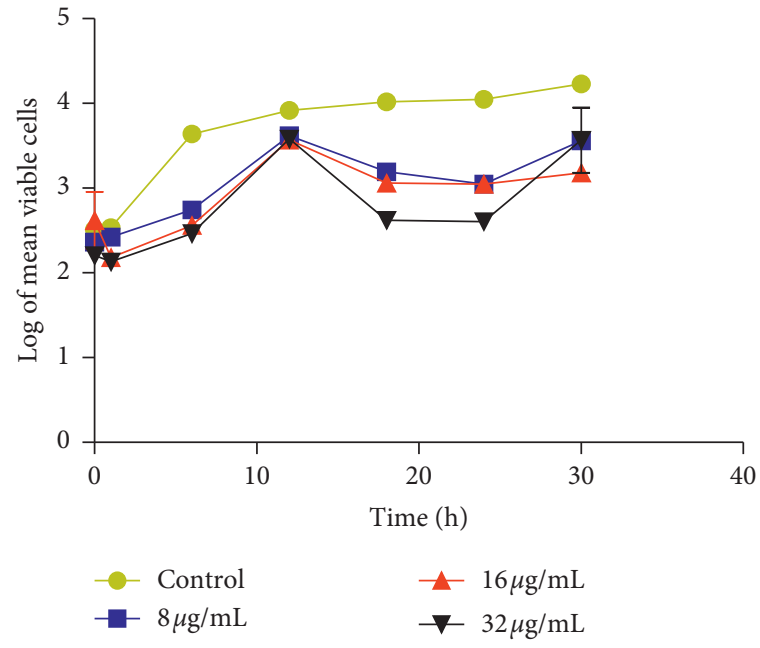

(a)

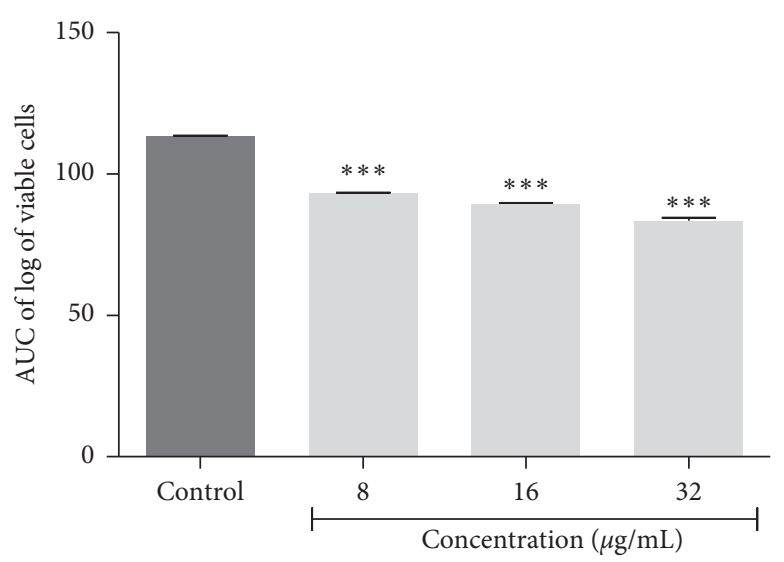

(b)

FIgURe 4: Time-kill kinetics of plumbagin against Pseudomonas aeruginosa. (a) Time-kill kinetics curve and (b) AUC of time-kill kinetics. AUC: area under the curve. $n=3$; values are mean \pm SEM. ${ }^{* * *} p<0.0001$ (one-way ANOVA followed by Dunnett's post hoc test).

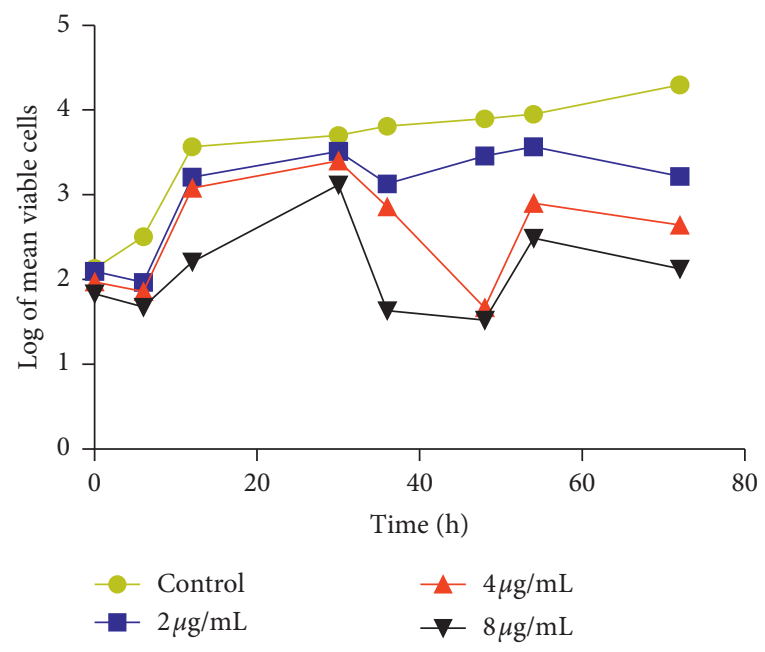

(a)

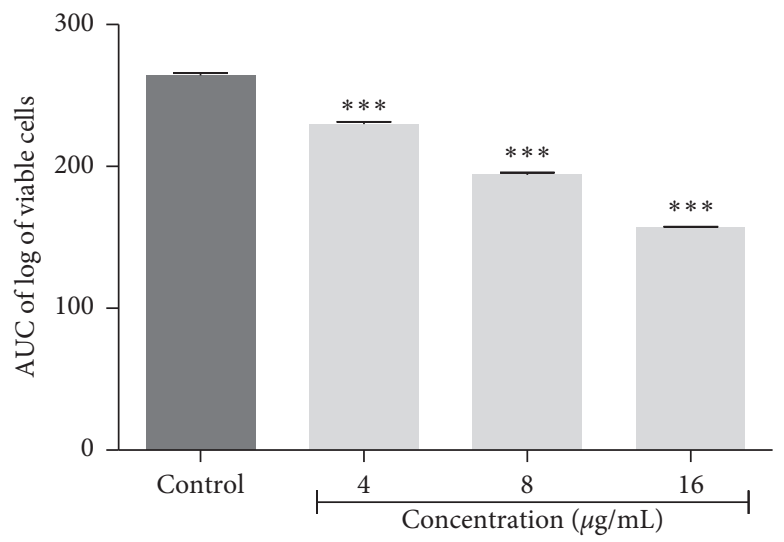

(b)

FIgURE 5: Time-kill kinetics of plumbagin against Candida albicans. (a) Time-kill kinetics curve and (b) AUC of time-kill kinetics. AUC: area under the curve. $n=3$; values are mean \pm SEM. ${ }^{* * *} p<0.0001$ (one-way ANOVA followed by Dunnett's post hoc test).

positive control, and biofilm formed in the absence of antimicrobial agent was used as negative control. Plumbagin demonstrated maximum inhibition against Escherichia coli at $128 \mu \mathrm{g} / \mathrm{mL}$ with $85 \%$ inhibition of biofilm formation, followed by $64 \mu \mathrm{g} / \mathrm{mL}, 32 \mu \mathrm{g} / \mathrm{mL}$, and $16 \mu \mathrm{g} / \mathrm{mL}$ with $82 \%, 78 \%$, and $72 \%$ inhibitory effects, respectively. Inhibition against Pseudomonas aeruginosa biofilm formation was recorded for ciprofloxacin at $128 \mu \mathrm{g} / \mathrm{mL}$ with $77 \%$ inhibition, followed by $64 \mu \mathrm{g} / \mathrm{mL}, 32 \mu \mathrm{g} / \mathrm{mL}$, and $16 \mu \mathrm{g} /$ $\mathrm{mL}$ with $72 \%, 68 \%$, and $64 \%$ inhibition, respectively. Plumbagin showed inhibition of $S$. aureus biofilm formation at $128 \mu \mathrm{g} / \mathrm{mL}$ with $66 \%$ inhibition followed by $64 \mu \mathrm{g} /$ $\mathrm{mL}$ and $32 \mu \mathrm{g} / \mathrm{mL}$ with $60 \%$ and $56 \%$, inhibitions, respectively. Inhibition against $K$. pneumoniae biofilm formation was recorded for plumbagin at $128 \mu \mathrm{g} / \mathrm{mL}$ with $52 \%$ inhibition followed by $64 \mu \mathrm{g} / \mathrm{mL}$ with $51 \%$ inhibition (Figure 6).

Ciprofloxacin (positive control) showed maximum inhibition against $E$. coli biofilm formation at $128 \mu \mathrm{g} / \mathrm{mL}$ with $87 \%$ inhibition, followed by $64 \mu \mathrm{g} / \mathrm{mL}, 32 \mu \mathrm{g} / \mathrm{mL}$, and $16 \mu \mathrm{g} /$ $\mathrm{mL}$ with $86 \%, 83 \%$, and $79 \%$ inhibition, respectively. Inhibition against $P$. aeruginosa biofilm formation was recorded at $128 \mu \mathrm{g} / \mathrm{mL}$ with $81 \%$ inhibition, followed by $64 \mu \mathrm{g} / \mathrm{mL}, 32 \mu \mathrm{g} / \mathrm{mL}$, and $16 \mu \mathrm{g} / \mathrm{mL}$ with $76 \%, 71 \%$, and $65 \%$ inhibitions, respectively. Inhibition against $S$. aureus biofilm formation was recorded at $128 \mu \mathrm{g} / \mathrm{mL}$ with $62 \%$ inhibition followed by $64 \mu \mathrm{g} / \mathrm{mL}$ with $50 \%$. Inhibition against $K$. pneumoniae biofilm formation for ciprofloxacin was recorded at $128 \mu \mathrm{g} / \mathrm{mL}$ with $53 \%$ followed by $64 \mu \mathrm{g} / \mathrm{mL}$ with $52 \%$ inhibition (Figure 6). 


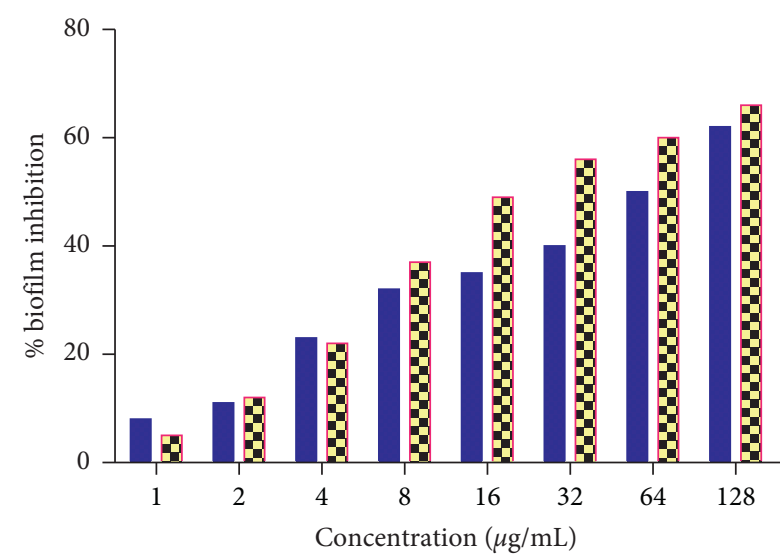

Ciprofloxacin Dlumbagin

(a)

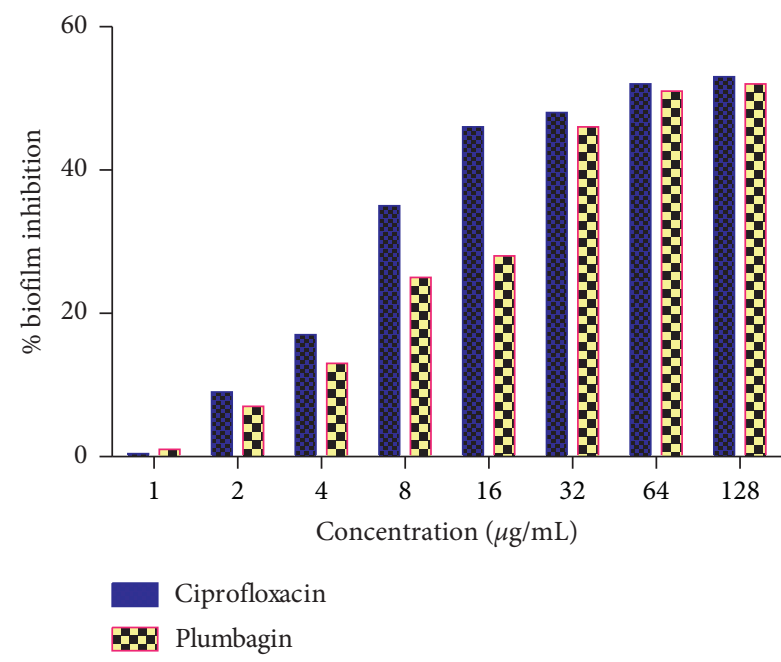

(c)

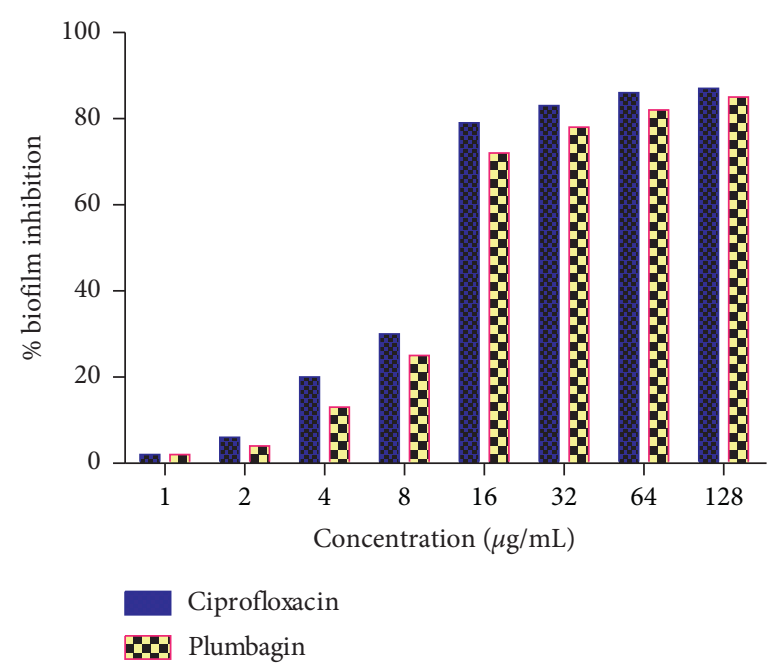

(b)

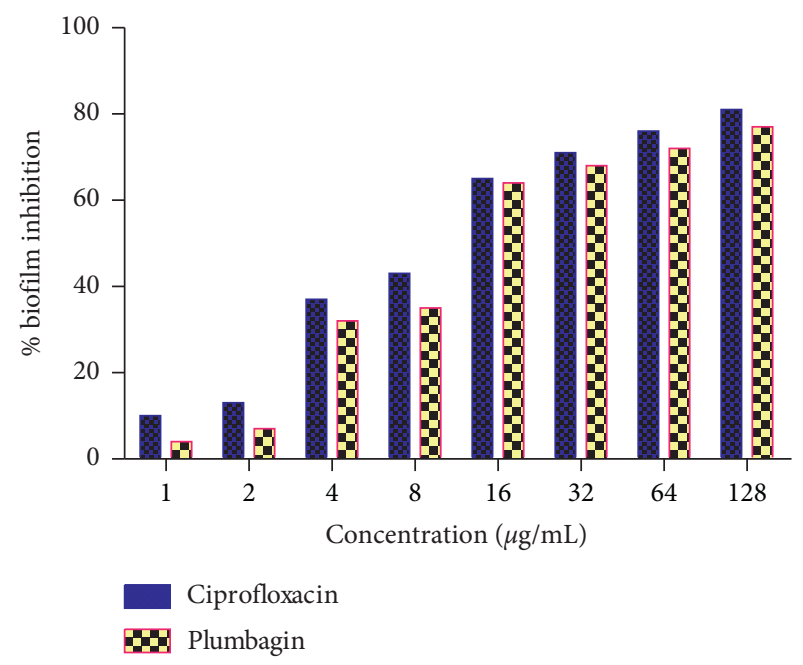

(d)

Figure 6: Percentage inhibition effect of plumbagin and ciprofloxacin (positive control) on biofilm formation by biofilm forming organisms: (a) Staphylococcus aureus, (b) Escherichia coli, (c) Klebsiella pneumoniae, and (d) Pseudomonas aeruginosa.

\section{Conclusion}

Plumbagin $(2.7 \mathrm{~g})$ was successfully isolated from $2.4 \mathrm{~kg}$ of the powdered roots of Plumbago zeylanica by column chromatography of the ethyl acetate extract. Its identity was confirmed using physical properties and spectroscopic methods. Plumbagin demonstrated activity against Staphylococcus aureus ATCC 25923, Escherichia coli ATCC 25922, Klebsiella pneumonia ATCC 10031, Pseudomonas aeruginosa ATCC 27853, and Candida albicans ATCC 10231 with MICs of $0.5,8,2,8$, and $4 \mu \mathrm{g} / \mathrm{mL}$, respectively. Plumbagin successfully modulated activities of ciprofloxacin, amoxicillin, ampicillin, and ketoconazole, implying that it has the potential to be developed as a drug candidate for use alongside commercial antimicrobials in combination therapies, in drug discovery and development to combat the menace of antimicrobial resistance. Plumbagin has also been established through a time-kill kinetics study to be bacteriostatic and fungistatic. Plumbagin demonstrated strong biofilm inhibition at concentrations of 128,64 , and $32 \mu \mathrm{g} / \mathrm{mL}$, so as the reference drug ciprofloxacin, suggesting its potential in the fight against antimicrobial resistance known to result to a greater extent from biofilm formation by the panel of organisms involved.

\section{Data Availability}

The data used to support the findings of this study are available at the Department of Pharmaceutical Chemistry, KNUST, Kumasi.

\section{Conflicts of Interest}

The authors declare that there are no conflicts of interest regarding the publication of this study. 


\section{Acknowledgments}

We wish to acknowledge the input and dedication of all authors towards this study. We are grateful to Mr. Francis Amankwah of the Microbiology Section, Department of Pharmaceutics, Faculty of Pharmacy and Pharmaceutical Sciences, KNUST, Kumasi, Ghana, for his assistance during the study. We also acknowledge Dr. Yaw Duah-Boakye and Dr. Cornelius Dodoo at the Microbiology Section, Department of Pharmaceutics KNUST, Kumasi and University of Health and Allied Sciences, Ho, respectively, for proofreading the manuscript.

\section{References}

[1] C. L. Ventola, "The antibiotic resistance crisis: part 1: causes and threats," $P$ \& T: A Peer-Reviewed Journal for Formulary Management, vol. 40, no. 4, pp. 277-283, 2015.

[2] WHO, WHO Report on Antimicrobial Resistance-A Global Epidemic, World Health Organization, Geneva, Switzerland, 2010.

[3] W. Kofie, C. D. Amengor, and R. K. Adosraku, "Synthesis and evaluation of antimicrobial properties of Azo dyes," International Journal of Pharmaceutical Sciences, vol. 7, no. 4, pp. 398-401, 2014.

[4] K. Ledingham, S. Hinchliffe, F. Thomas, and G. Thomson, "Antibiotic resistance: using a cultural contexts of health approach to address a global health challenge," Policy Briefing No 2, World Health Organisation, Geneva, Switzerland, 2019.

[5] S. O. Dapaah, C. Agyare, Y. D. Boakye, and T. Appiah, "Modulatory effects of Hilleria latifolia and Laportea ovalifolia on activity of selected antibiotics," Journal of Medicinal Plants Research, vol. 10, no. 25, pp. 360-366, 2016.

[6] T. Sibanda and A. I. Okoh, "The challenges of overcoming antibiotic resistance: plant extracts as potential sources of antimicrobial and resistance modifying agents," African Journal of Biotechnology, vol. 6, no. 25, pp. 2886-2896, 2007.

[7] M. Balouiri, M. Sadiki, and S. K. Ibnsouda, "Methods for in vitro evaluating pharmaceutical analysis; a review," Journal of Pharmaceutical Analysis, vol. 1, no. 11, pp. 1-9, 2016.

[8] G. Priya, S. Arpita, S. Padmani, D. Akshay, D. Manash, and B. Surajt, "Potentiation of antibiotic against Pseudomonas aeruginosa biofilm: a study with plumbagin and gentamicin," Journal of Applied Microbiology, vol. 123, no. 1, pp. 246-261, 2017.

[9] L. Clontz, "Biofilm inhibition: the use of a marine alkaloid derivative in the prevention of clinically-relevant biofilms," Journal of Microbiology \& Experimentation, vol. 6, no. 5, pp. 206-214, 2018.

[10] Y. D. Mandavkar and S. S. Jalalpure, "A comprehensive review on Plumbago zeylanica Linn," African Journal of Pharmacy and Pharmacology, vol. 5, no. 25, pp. 2738-2747, 2011.

[11] J. E. Adaeze, E. E. Dishan, and I. O. Tella, "Plants species diversity along river benue bank under the influence of siltation and solid waste effluents, Adamawa state, Nigeria," Open Access Library Journal, vol. 4, no. 12, 2017.

[12] S. K. Sandur, H. Ichikawa, G. Sethi, K. S. Ahn, and B. B. Aggarwal, "Plumbagin (5-hydroxy-2-methyl-1,4-naphthoquinone) suppresses NF- $\kappa \mathrm{B}$ activation and NF- $\kappa \mathrm{B}$-regulated gene products through modulation of p65 and $\mathrm{I} \kappa \mathrm{B} \alpha$ kinase activation, leading to potentiation of apoptosis induced by cytokine and chemotherapeutic agents," Journal of Biological Chemistry, vol. 281, no. 25, pp. 17023-17033, 2006.
[13] K. Annan, R. A. Dickson, and A. Y. Mensah, "Acaricidal effect of Plumbago zeylanica Linn against Amblyoma variegatum," Pharmacognosy Journal, vol. 1, no. 3, pp. 190-194, 2009.

[14] W. Sumsakul, T. Plengsuriyakarn, W. Chaijaroenkul, V. Viyanant, J. Karbwang, and K. Na-Bangchang, "Antimalarial activity of plumbagin in vitro and in animal models," BMC Complementary and Alternative Medicine, vol. 14, no. 1, pp. 1-9, 2014.

[15] S.-X. Wang, J. Wang, J.-B. Shao, W.-N. Tang, and J.-Q. Zhong, "Plumbagin mediates cardioprotection against myocardial ischemia/reperfusion injury through nrf-2 signaling," Medical Science Monitor, vol. 22, pp. 1250-1257, 2016.

[16] M. Tan, Y. Liu, X. Luo, Z. Chen, and H. Liang, "Antioxidant activities of plumbagin and its $\mathrm{Cu}$ (II) complex," Bioinorganic Chemistry and Applications, vol. 2011, Article ID 898726, 5 pages, 2011.

[17] S. Padhye, P. Dandawate, M. Yusufi, A. Ahmad, and F. H. Sarkar, "Perspective on medicinal properties of plumbagin and its analogue," ARPN Journal of Engineering and Applied Sciences, vol. 10, no. 7, pp. 3067-3071, 2015.

[18] S. R. Paiva, R. M. Figueiredo, V. T. Aragão, and M. A. C. Kaplan, "Antimicrobial activity in-vitro of plumbagin isolated from Plumbago species," Memórias do Instituto Oswaldo Cruz, vol. 98, no. 7, pp. 951-961, 2003.

[19] L. Lai, J. Liu, D. Zhai et al., "Plumbagin inhibits tumour angiogenesis and tumour growth through the Ras signalling pathway following activation of the VEGF receptor-2," British Journal of Pharmacology, vol. 165, no. 4b, pp. 1084-1096, 2012.

[20] J. Kovač, N. Gavarić, F. Bucar, and S. S. Možina, "Antimicrobial and resistance modulatory activity of Alpinia katsumadai seed phenolic extract, essential oil and post-distillation extract," Food Technology and Biotechnology, vol. 52, no. 2, pp. 248-254, 2014.

[21] F. Adu, S. Gbedema, and K. Annan, "Antimicrobial and resistance modulatory activities of Corynanthe pachyceras," Pharmacognosy Research, vol. 1, no. 5, pp. 280-284, 2009.

[22] A. Francis, H. S. George, A. Christian, A. A. John, E. B. Vivian, and N. David, "Influence of methanol fruit and leaf extracts of Myristica fragrans (Myristicaceae) on the activity of some antibiotics," African Journal of Microbiology Research, vol. 8, no. 19, pp. 1982-1986, 2014.

[23] T. Appiah, Y. D. Boakye, and C. Agyare, "Antimicrobial activities and time-kill kinetics of extracts of selected Ghanaian mushrooms," Evidence-based Complementary and Alternative Medicine, vol. 2017, Article ID 4534350, 15 pages, 2017.

[24] F. Haque, M. Alfatah, K. Ganesan, and S. Bhattacharyya, "Inhibitory effect of sophorolipid on Candida albicans biofilm formation and hyphal growth," Scientific Reports, vol. 6, p. $23575,2016$. 


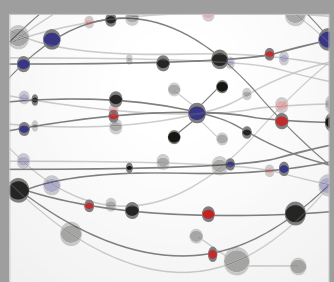

The Scientific World Journal
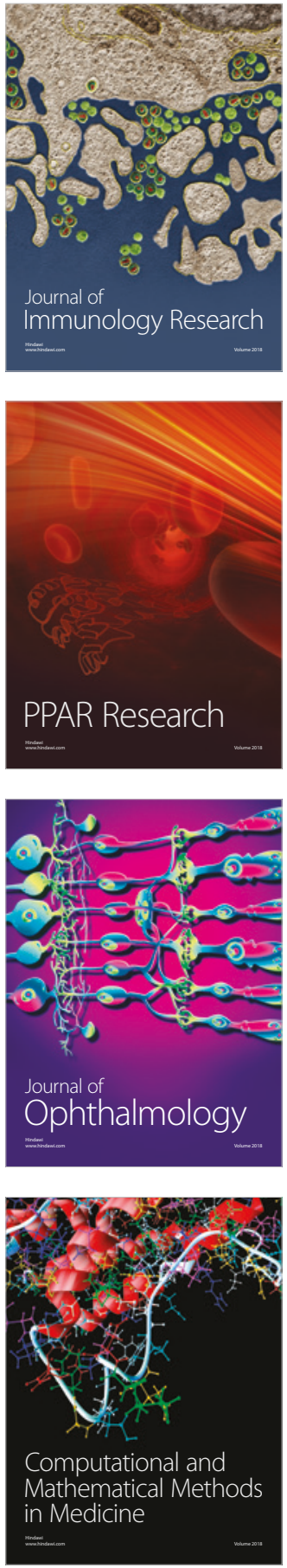

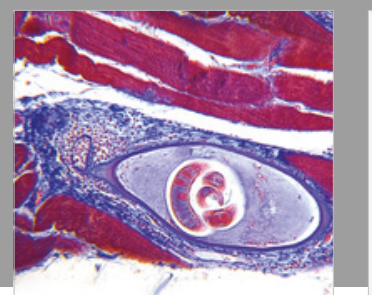

Gastroenterology Research and Practice

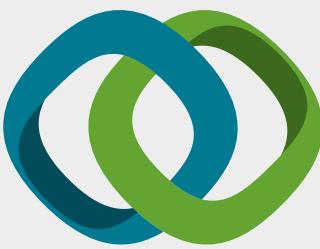

\section{Hindawi}

Submit your manuscripts at

www.hindawi.com
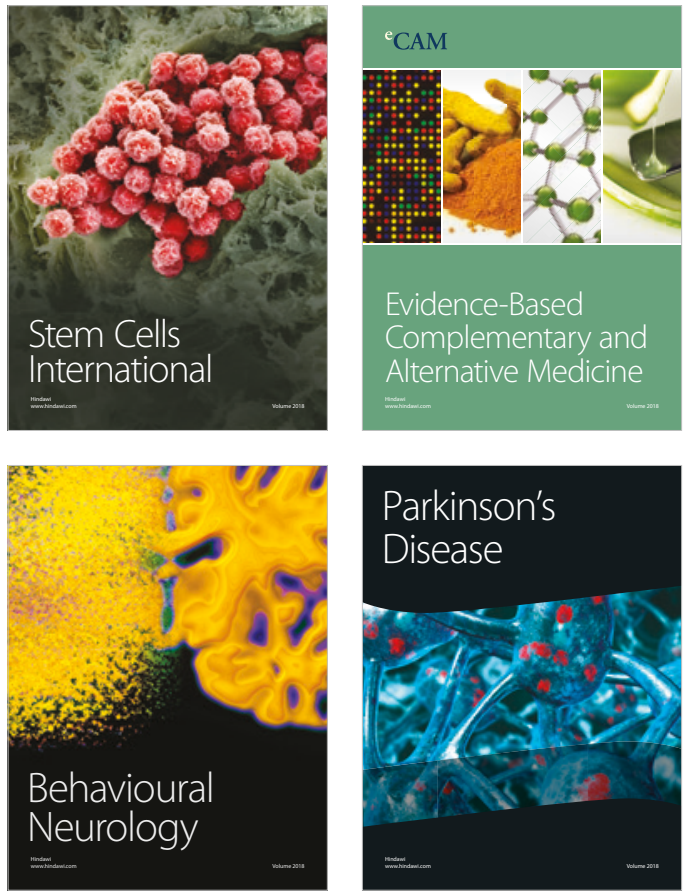

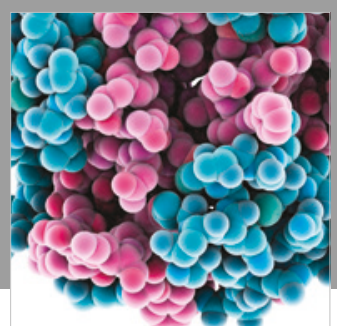

ournal of

Diabetes Research

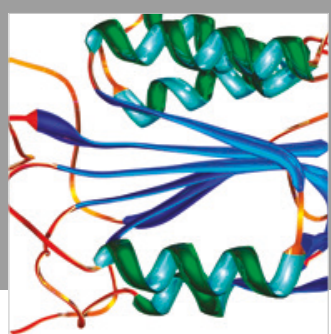

Disease Markers
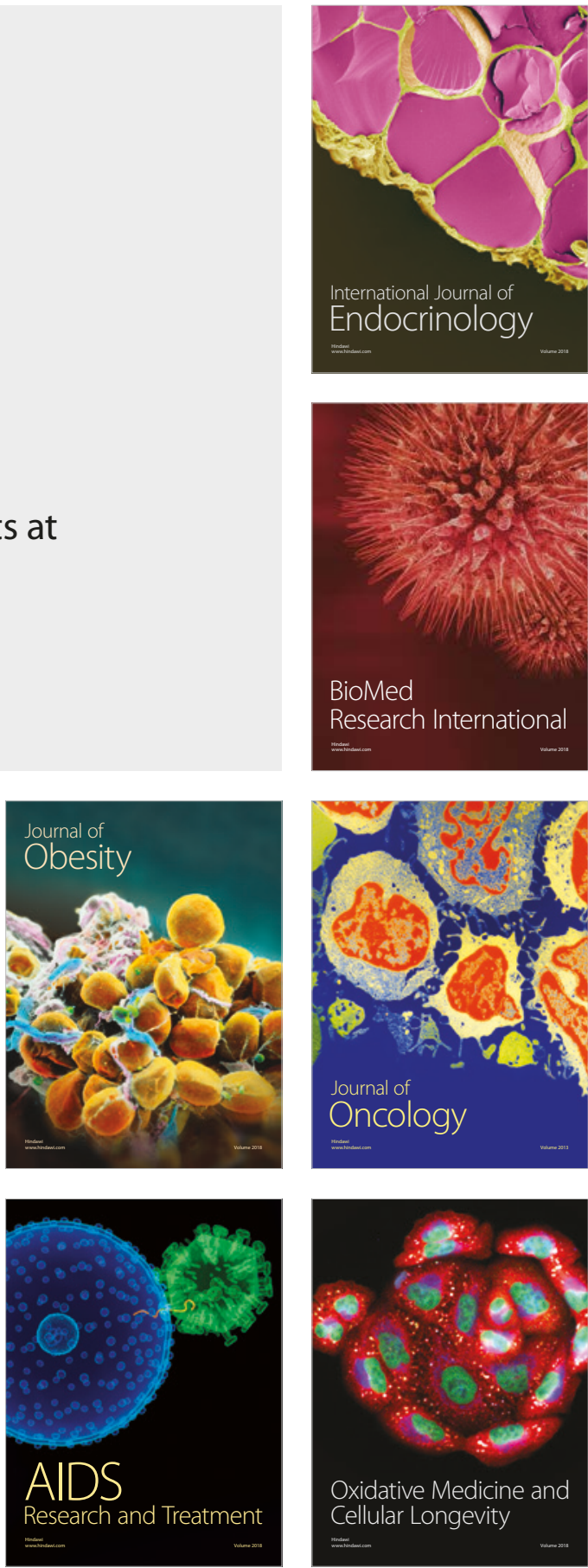